Homology, Homotopy and Applications, vol.20(2), 2018, pp.79-103

\title{
A SEIFERT-VAN KAMPEN THEOREM IN NON-ABELIAN ALGEBRA
}

\author{
MATHIEU DUCKERTS-ANTOINE AND TIM VAN DER LINDEN \\ (communicated by George Janelidze)
}

\begin{abstract}
We prove a variation on the Seifert-van Kampen theorem in a setting of non-abelian categorical algebra, providing sufficient conditions on a functor $F$, from an algebraically coherent semiabelian category with enough projectives to an almost abelian (= Raikov semiabelian) category, for the preservation of pushouts of split monomorphisms by the left derived functor of $F$.
\end{abstract}

\section{Introduction}

Seifert and Van Kampen's famous theorem on the fundamental group of a union of two spaces $[67,72]$ has been sharpened and extended to other contexts in many ways $[17,40,56,20,68,19,75,21,69]$. Let us here recall the following elementary version. Consider a commutative square of pointed topological spaces and base-pointpreserving continuous maps as on the left,
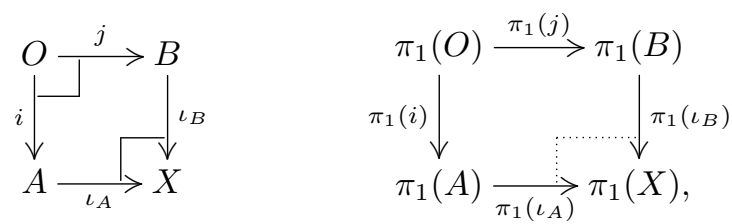

where $A, B$ and $O$ are open, path-connected subspaces of $X$, and

- the square is a pushout, that is, $X=A+_{O} B$ is the amalgamated sum of $A$ and $B$ over $O$;

- the square is a pullback, that is, $O=A \times_{X} B=A \cap B$ is the intersection of $A$ and $B$ in $X$.

Then the square on the right obtained by applying the fundamental group functor is a pushout in the category of groups.

The aim of our article is to present a variation on this result, valid in a rather general categorical-algebraic context. We prove a Seifert-van Kampen theorem in a non-additive setting, giving sufficient conditions on a functor $F: \mathscr{C} \rightarrow \mathscr{X}$ from an

Received January 24, 2017; published on May 2, 2018.

2010 Mathematics Subject Classification: 18G10, 18G50, 18A40, 20J99, 55N99.

Key words and phrases: derived functor, fundamental group, homology coproduct theorem, categorical Galois theory, algebraically coherent semi-abelian category.

Article available at http://dx.doi.org/10.4310/HHA.2018.v20.n2.a5

Copyright (C) 2018, Mathieu Duckerts-Antoine and Tim Van der Linden. Permission to copy for private use granted. 
algebraically coherent [24] semi-abelian [50] category $\mathscr{C}$ to an almost abelian [66] (also called Raikov semiabelian [62] or biregular additive) category $\mathscr{X}$ for its fundamental group [48] functor $\pi_{1}^{F}$ to preserve pushouts of split monomorphisms. We shall recall the definitions of these concepts in sections 2 and 3. Let us just mention now that here $\mathscr{C}$ may, for instance, be the category of groups, rings, crossed modules, or Lie algebras over a field; while $\mathscr{X}$ may for example be a category of modules, Banach spaces, or locally compact abelian groups. When $\mathscr{X}$ is abelian, this fundamental group functor $\pi_{1}^{F}$ is precisely the classical left derived functor $\mathscr{L}_{1} F$ of $F$.

\subsection{Motivation}

This work is part of a long-term project aiming to understand group (co)homology from a categorical-algebraic perspective. Within this larger context, our interest in a general Seifert-van Kampen theorem is twofold. On the one hand, through an analysis of this theorem we are studying which aspects of group (co)homology are typical for groups, and which others arise for purely formal reasons, so that a categorical argument suffices to comprehend and apply them in other settings. On the other hand, such an analysis helps us see what the needs of homological algebra tell us about categories of non-abelian algebraic structures: we are asking ourselves what are the right conditions on a category for a given result in homological algebra to hold. These questions may have unexpected answers - for instance, the one given here which involves a seemingly unrelated technical condition called algebraic coherence by Cigoli, Gray and Van der Linden [24].

\subsection{A special case: preservation of binary sums}

In the special case where the pushout under consideration is a coproduct, our Seifert-van Kampen theorem may be seen as a non-abelian version of a fact which is well known in the abelian case. Indeed, for any additive functor $F: \mathscr{C} \rightarrow \mathscr{X}$ between abelian categories $\mathscr{C}$ and $\mathscr{X}$ where $\mathscr{C}$ has enough projectives, the left derived functors $\mathscr{L}_{n} F: \mathscr{C} \rightarrow \mathscr{X}$ are additive [23]. In other words, if $F$ preserves binary coproducts, then each derived functor of $F$ does. One goal of the article is to explore how this extends to a general homology coproduct theorem in the sense of Barr and Beck [3], valid in a non-abelian algebraic context.

\subsection{Known non-abelian results}

Of course several instances of a non-abelian homology coproduct theorem can already be found in the literature. For example, given any two groups $X, Y$ and any $n \geqslant 0$, there is the isomorphism

$$
H_{n+1}(X+Y, \mathbb{Z}) \cong H_{n+1}(X, \mathbb{Z}) \oplus H_{n+1}(Y, \mathbb{Z})
$$

describing the integral homology of the free product $X+Y$ as the direct sum of the homologies of $X$ and $Y$. See $[2,3,74]$ for an algebraic proof of this result, which may also be obtained by using the corresponding isomorphism for topological spaces (via the classical Seifert-van Kampen theorem). This may be reinterpreted as an isomorphism

$$
\mathscr{L}_{n} F(X+Y) \cong \mathscr{L}_{n} F(X) \oplus \mathscr{L}_{n} F(Y)
$$


where $F$ is the abelianisation functor ab: $\mathrm{Gp} \rightarrow \mathrm{Ab}$, which sends a group $X$ to its abelianisation $\operatorname{ab}(X)=X /[X, X]$.

Similar results hold, for instance, for rational homotopy of topological spaces [26] and for homology of graded associative algebras [5, 44, 42]; see also Mac Lane [54]. Barr and Beck give several examples of homology coproduct theorems in the context of comonadic homology [3].

\subsection{A negative result}

Attempting to find a general non-additive homology coproduct theorem for $\mathscr{L}_{n} F$, one is immediately confronted with the following counterexample, which was obtained by Johnson [51] as a test of the homology theory for varieties of groups established by Leedham-Green [53]. If $F: \mathrm{Nil}_{2} \rightarrow \mathrm{Ab}$ is the reflector from the category of groups of nilpotency class at most two to the category of abelian groups, so the restriction to $\mathrm{Nil}_{2}$ of the abelianisation functor considered above, then

$$
\mathscr{L}_{2} F\left(C_{2}+C_{2}\right)=C_{2} \oplus C_{2} \oplus C_{2} \not C_{2} \oplus C_{2}=\mathscr{L}_{2} F\left(C_{2}\right) \oplus \mathscr{L}_{2} F\left(C_{2}\right),
$$

where $C_{2}$ is the cyclic group of order two.

We have to conclude that a general homology coproduct theorem applicable to the functor ab: $\mathrm{Nil}_{2} \rightarrow$ Ab cannot exist. This leaves open essentially only two courses of investigation:

(1) to study the case $n=1$ in a setting wide enough to include the variety of groups of nilpotency class at most two;

(2) to study the situation for general $n$, but in a much more restricted context which includes groups while ruling out the category $\mathrm{Nil}_{2}$.

In the present paper we focus on (1) in order to prove a general homology coproduct theorem for $\mathscr{L}_{1} F$ as a particular case of an algebraic Seifert-van Kampen theorem. We hope to come back to (2) in future work; for now, in Section 5 we make some further remarks about this problem.

\subsection{Our setting}

The situation we consider in this paper is that of a regular epi-reflection

$$
\mathscr{C} \underset{\stackrel{\perp}{\stackrel{\perp}{\gtrless}}}{\stackrel{F}{\gtrless}} \mathscr{X},
$$

where $\mathscr{C}$ is an algebraically coherent [24] semi-abelian [50] category with enough regular projectives and $\mathscr{X}$ is an abelian category. It is this functor $F$ which we are going to derive - using simplicial resolutions [70] in the abstract case, or comonadic resolutions [3] when $\mathscr{X}$ is a variety of algebras. Alternatively, the standard Quillen model structure on the category of simplicial objects in $\mathscr{C}$ may be used $[61,71]$ : thus $\mathscr{L}_{1} F$ may be seen as Quillen's first derived functor.

\subsection{Some examples}

In Section 2 we shall recall the definitions of the above-mentioned categoricalalgebraic conditions. For the time being, let us just mention that any Orzech category of interest [60] is semi-abelian algebraically coherent, so that we might consider any reflector from such a variety $\mathscr{C}$ to an abelian subvariety $\mathscr{X}$ of $\mathscr{C}$. For instance, the 
reflector from groups to abelian groups, from groups of a certain nilpotency class to abelian groups, from crossed modules to abelian crossed modules, from Lie algebras over a field $\mathbb{K}$ to $\mathbb{K}$-vector spaces, from $G$-actions to $\mathbb{Z}[G]$-modules for a given group $G$ all fit into this setting. More examples will be given in Section 2 and Section 3.

\subsection{The fundamental group functor}

The proof of our result is based on Janelidze's categorical Galois theory [46, 47, 49, 9], with, in particular, the interpretation given in work of Duckerts, Everaerts, Goedecke, and Van der Linden $[48,36,32,28]$ of the derived functor $\mathscr{L}_{1} F$ as a fundamental group functor $\pi_{1}^{F}$. This leads to a slightly more general result, which remains valid even when $\mathscr{X}$ is not abelian, but only almost abelian in the sense of Rump [66] or semiabelian in the sense of Raikov [62].

In the given context, there is the concept of an extension (= a regular epimorphism in $\mathscr{C}$ ) and that of a normal extension (defined with respect to the functor $F: \mathscr{C} \rightarrow \mathscr{X})$. It turns out that the inclusion $\operatorname{NExt}(\mathscr{C}) \rightarrow \operatorname{Ext}(\mathscr{C})$ of the category of normal extensions into the category of extensions admits a left adjoint. This left adjoint functor is called the normalization functor relative to $F$ and is denoted $F_{1}: \operatorname{Ext}(\mathscr{C}) \rightarrow \operatorname{NExt}(\mathscr{C})$.

For instance, an extension in the category of groups is just a surjective group homomorphism. If now $F$ is the abelianisation functor $\mathrm{ab}: \mathrm{Gp} \rightarrow \mathrm{Ab}$, then a normal extension is the same thing as a central extension: a surjective group homomorphism $f: X \rightarrow Y$ for which the elements of the kernel $K$ commute with all the elements of $X$. The functor $F_{1}$ takes an extension $f: X \rightarrow Y$ and sends it to the quotient $F_{1}(f): X /[K, X] \rightarrow Y$. Here the commutator $[K, X]$ is generated by the elements of the form $k x k^{-1} x^{-1}$ where $k \in K$ and $x \in X$.

When it exists, the fundamental group functor $\pi_{1}^{F}: \mathscr{C} \rightarrow \mathscr{X}$ relative to the functor $F$ is defined as the pointwise right Kan extension [55]

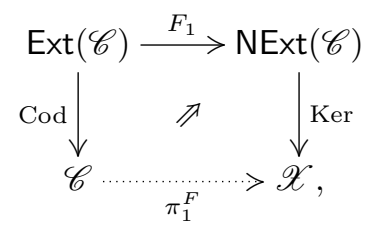

where Cod sends an extension $f: X \rightarrow Y$ to its codomain $Y$ and Ker sends a (normal) extension $g$ to its kernel $\operatorname{Ker}(g)$. If $\mathscr{C}$ has enough projectives and $\mathscr{X}$ is abelian, then the functor $\pi_{1}^{F}$ corresponds to the first left derived functor of $F$ obtained via simplicial resolutions: we have an isomorphism $\pi_{1}^{F} \cong \mathscr{L}_{1} F$, where the derived functor on the right is as in $[3,70]$. In the case of abelianisation of groups, for instance, we see that $\pi_{1}^{F}(X) \cong H_{2}(X, \mathbb{Z})$, the second integral homology group.

\subsection{The result}

We prove that the first fundamental group functor $\pi_{1}^{F}: \mathscr{C} \rightarrow \mathscr{X}$ preserves pushouts of split monomorphisms, so that - when the derived functor in question is a fundamental group functor - for any pushout of split monomorphisms $i$ and $j$ in $\mathscr{C}$ as on 
the left,
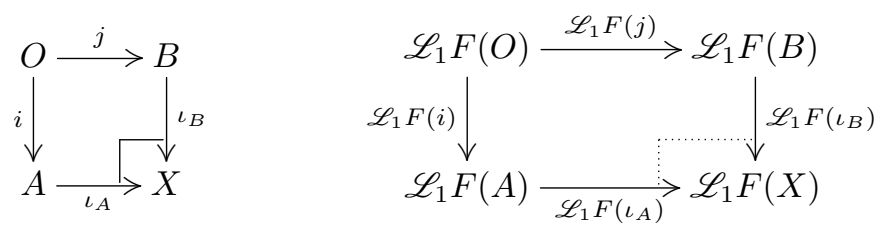

the resulting square on the right is a pushout in $\mathscr{X}$. In particular, by taking $O=0$ it follows that for any $A$ and $B$ in $\mathscr{C}$,

$$
\mathscr{L}_{1} F(A+B) \cong \mathscr{L}_{1} F(A) \oplus \mathscr{L}_{1} F(B) .
$$

\subsection{Structure of the text}

In Section 2 we recall some basic terminology and sketch the context in which we shall be working. Section 3 recalls Galois-theoretic notions and results concerning the fundamental group functor. Most of the real work is done in Section 4 where some technical lemmas having to do with jointly strongly epimorphic pairs of arrows are proved. Section 5 contains our main result, the Seifert-van Kampen theorem. We obtain two versions: first, in Theorem 5.1, we consider the case where $\mathscr{X}$ is an almost abelian category [66], and the left adjoint reflector $F$ preserves pullbacks of split epimorphisms along regular epimorphisms; then we find that $\pi_{1}^{F}$ preserves pushouts of split monomorphisms. We next restrict the setting to the case where $\mathscr{X}$ is abelian and $F$ is a Birkhoff reflector. Then $\pi_{1}^{F}$ is a genuine left derived functor of $F$, and we obtain Theorem 5.2.

\section{Acknowledgments}

This work was supported by the FCT-Fundação para a Ciência e a Tecnologia under the grant number SFRH/BPD/98155/2013, and by the Centre for Mathematics of the University of Coimbra-UID/MAT/00324/2013, funded by the Portuguese Government through FCT/MEC and co-funded by the European Regional Development Fund through the Partnership Agreement PT2020.

The first author wishes to thank the Institut de Recherche en Mathématique et Physique - IRMP for its kind hospitality during his stays in Louvain-la-Neuve.

The second author is a Research Associate of the Fonds de la Recherche Scientifique-FNRS. He would like to thank the CMUC for its kind hospitality during his stays in Coimbra.

\section{Terminology}

Semi-abelian categories were introduced by Janelidze, Márki and Tholen [50] in order to unify several historically important approaches to a categorical framework for the study of homological algebra of non-abelian algebraic objects (e.g., [41, 45, 1 , 10]). Next to all abelian categories, any variety of $\Omega$-groups in the sense of Higgins [41] is an example. So for a variety of algebras to be semi-abelian, it suffices that amongst the operations and identities of the theory, there is a unique constant and a group operation. This easily provides us with many examples such as groups, (non-unitary) 
rings, associative algebras, Lie algebras, crossed modules, etc. Further examples of a different kind include loops [7], Heyting semilattices [52], cocommutative Hopf algebras over a field of characteristic zero [38], and the dual of the category of pointed sets [13].

\subsection{Formal definition}

A category is semi-abelian [50] when it is pointed, exact and protomodular with binary coproducts. Let us briefly recall the definitions of these notions and their basic properties.

Let us first note that in presence of the other axioms, the existence of binary coproducts suffices for the category to be finitely complete and finitely cocomplete. So we freely use finite limits and finite colimits as needed.

Pointed means that there is a zero object, written 0: an initial object which is also terminal. Often, the zero object is a one-element algebra. A morphism which factors through the zero object is called a zero map; given any two objects $A$ and $B$, a unique such $0: A \rightarrow B$ exists. For any morphism $f: A \rightarrow B$, the coequaliser of $f$ and 0 is called the cokernel of $f$, and written $\operatorname{coker}(f): B \rightarrow \operatorname{Coker}(f)$. Dually, we write $\operatorname{ker}(f): \operatorname{Ker}(f) \rightarrow A$ for the kernel of $f$, the equalizer of $f$ and 0 . A short exact sequence is a pair of morphisms $(k, f)$ where $k=\operatorname{ker}(f)$ and $f=\operatorname{coker}(k)$; this situation is usually pictured as a sequence

$$
0 \longrightarrow K \triangleright^{k} \longrightarrow A \stackrel{f}{\longrightarrow} B \longrightarrow 0 .
$$

A morphism which is the cokernel of some morphism is called a normal epimorphism, and a morphism which is the kernel of some morphism is called a normal monomorphism. In many varieties of algebras, normal monomorphisms correspond to inclusions of (suitably defined) ideals. The cokernel of a homomorphism $f: A \rightarrow B$ may then be obtained as the quotient of $B$ by the normal closure of the image of $f$. It is important to notice that not all monomorphisms need to be normal: indeed, non-normal subgroups exist.

The concept of an image itself is formalized as follows. A morphism is called a regular epimorphism when it is the coequaliser of some parallel pair of arrows. In any variety of algebras these are precisely the surjective homomorphisms - unlike the epimorphisms, which in this context turn out to play a lesser role. Obviously, by definition, any normal epimorphism is a regular epimorphism; conversely, in a semiabelian category, the two concepts may be seen to coincide. A category is regular [1] if it is finitely complete, every morphism can be factorized as a regular epimorphism followed by a monomorphism, and regular epimorphisms are stable under pullbacks. The monomorphism in this (essentially unique) factorization of a morphism $f$ is called the image of $f$.

A relation $\left(R, d_{1}, d_{2}\right)$ from $A$ to $B$ is a subobject of the product $A \times B$, represented by a monomorphism $\left\langle d_{1}, d_{2}\right\rangle: R \rightarrow A \times B$. Relations are naturally ordered by inclusion. In the context of a regular category, it is possible to consider the composite of two relations, and then an internal equivalence relation on an object $A$ may be defined as a relation $R$ which is reflexive $\left(\Delta_{A} \leqslant R\right.$, where $\left.\Delta_{A}=\left\langle 1_{A}, 1_{A}\right\rangle: A \rightarrow A \times A\right)$, symmetric $\left(R^{\mathrm{op}} \leqslant R\right.$, where $\left.\left(R^{\mathrm{op}}, d_{1}^{\mathrm{op}}, d_{2}^{\mathrm{op}}\right)=\left(R, d_{2}, d_{1}\right)\right)$ and transitive $(R \circ R \leqslant R)$. The kernel relation or kernel pair $\left(\mathrm{Eq}(f), \operatorname{pr}_{1}^{f}, \operatorname{pr}_{2}^{f}\right)$ of a morphism $f: A \rightarrow B$, which 
is the pullback of $f$ along itself, considered as a subobject of $A \times A$, is always an equivalence relation on $A$. The converse need not hold in general. Whence the definition of a (Barr) exact [1] category, which is regular and such that every internal equivalence relation is the kernel relation of some morphism. It is well known that all varieties of algebras are Barr exact.

A pointed category with finite limits is (Bourn) protomodular [10] when the Split Short Five Lemma holds. This means that whenever we have a diagram

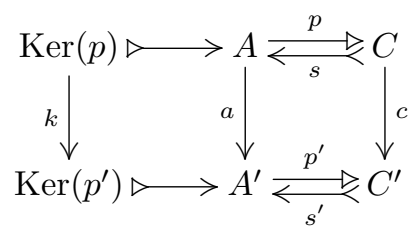

in which the three squares commute while $p \circ s=1_{C}$ and $p^{\prime} \circ s^{\prime}=1_{C^{\prime}}$, if the morphisms $k$ and $c$ are isomorphisms then $a$ is an isomorphism.

A category which is pointed, regular and protomodular is said to be homological [7]. In a homological category, the basic lemmas of homological algebra, such as the Short Five Lemma, the $3 \times 3$ Lemma and the Snake Lemma hold. Such a category is Barr exact if and only if the image of a composite $p \circ k$, where $p$ is a normal epimorphism and $k$ is a normal monomorphism, is again a normal monomorphism. Then it suffices that binary coproducts exist for the category to be semi-abelian.

Examples are given below in 2.6. For now, let us just recall the following

Lemma 2.1 ([22, Theorem 5.7]). In a semi-abelian category, let us consider a square of regular epimorphisms

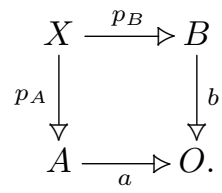

The comparison morphism $\left\langle p_{A}, p_{B}\right\rangle: X \rightarrow A \times_{O} B$ to the pullback of $a$ and $b$ is a regular epimorphism if and only if the square is a pushout.

\subsection{The category of points}

Protomodularity may be reformulated in more abstract terms as follows.

Let $\mathscr{C}$ be a category with pullbacks of split epimorphisms along any morphism. A point in $\mathscr{C}$ is a pair $(p: A \rightarrow C, s: C \rightarrow A)$ of morphisms in $\mathscr{C}$ such that $p \circ s=1_{C}$ : it is a split epimorphism $p$ with a chosen splitting $s$. (Equivalently, of course, a point $(p, s)$ may be considered as a split monomorphism $s$ with a chosen splitting $p$.) The category of points $\operatorname{Pt}(\mathscr{C})$ has as objects points in $\mathscr{C}$. Morphisms are natural transformations between those. More precisely, an arrow

$$
f:(p: A \rightarrow C, s: C \rightarrow A) \rightarrow\left(p^{\prime}: A^{\prime} \rightarrow C^{\prime}, s^{\prime}: C^{\prime} \rightarrow A^{\prime}\right)
$$

is a pair $f=\left(f_{1}: A \rightarrow A^{\prime}, f_{0}: C \rightarrow C^{\prime}\right)$ such that $p^{\prime} \circ f_{1}=f_{0} \circ p$ and $f_{1} \circ s=s^{\prime} \circ f_{0}$.

We write $\mathrm{Pt}^{2}(\mathscr{C})$ for the category $\operatorname{Pt}(\operatorname{Pt}(\mathscr{C}))$. Its objects are split epimorphisms of split epimorphisms with chosen splittings: squares such as (D) below. 
The forgetful codomain functor

$$
\text { Cod: } \operatorname{Pt}(\mathscr{C}) \rightarrow \mathscr{C}: \quad(p: A \rightarrow C, s: C \rightarrow A) \mapsto C, \quad f=\left(f_{1}, f_{0}\right) \mapsto f_{0}
$$

is a fibration (see [6] for instance) which is called the fibration of points. We write $\operatorname{Pt}_{X}(\mathscr{C})$ for the fibre over $X$, which is the subcategory of $\operatorname{Pt}(\mathscr{C})$ determined by the morphisms $f$ such that $\operatorname{Cod}(f)=1_{X}$. The objects of this category are called points over $X$ in $\mathscr{C}$.

For any morphism $f: A \rightarrow B$ in $\mathscr{C}$, pulling back a point $(p, s)$ along the morphism $f$ induces a change-of-base functor

$$
f^{*}: \mathrm{Pt}_{B}(\mathscr{C}) \rightarrow \mathrm{Pt}_{A}(\mathscr{C}) .
$$

It is not very difficult to see that the category $\mathscr{C}$ is protomodular if, and only if, all change-of-base functors $f^{*}$ are conservative, which means that they reflect isomorphisms.

When $\mathscr{C}$ is homological, there is [15] a concept of internal action which induces an equivalence between "split extensions" and "actions" - formally, this is the monadicity of the functor $\operatorname{Cod}: \operatorname{Pt}(\mathscr{C}) \rightarrow \mathscr{C}$. For us right now, it suffices to understand the following interpretation of this fact. Any point $(p, s)$ induces a split extension

$$
0 \longrightarrow K \triangleright \stackrel{\operatorname{ker}(p)}{\longrightarrow} A \stackrel{p}{\longleftarrow s} \rightleftharpoons C \longrightarrow 0 .
$$

This split extension corresponds to an internal action $\xi$ of $C$ on $K$ in the sense of [15] via a general semi-direct product construction: there is a split extension

$$
0 \longrightarrow K \stackrel{\operatorname{ker}\left(p_{\xi}\right)}{\longrightarrow} K \rtimes_{\xi} C \stackrel{p_{\xi}}{\gtrless} \underset{s_{\xi}}{\gtrless} C \longrightarrow 0,
$$

which turns out to be isomorphic to the given split extension.

In other words, a point $(p, s)$ over $C$ in $\mathscr{C}$ may be seen as some kind of a "nonabelian Beck module" [4] via the internal $C$-action it induces on the kernel $K$ of $p$. Protomodularity thus amounts to the condition that any morphism of $C$-actions which induces an isomorphism $K \rightarrow K^{\prime}$ on the underlying objects (= kernels) $K$ and $K^{\prime}$ necessarily is an isomorphism itself. This point of view may help better understanding the definition in Section 2.5 below.

\subsection{Epimorphisms}

A morphism $f: A \rightarrow B$ in a finitely complete category $\mathscr{C}$ is

- an extremal epimorphism if, for every commutative triangle as on the left with $m$ a monomorphism, $m$ is an isomorphism;
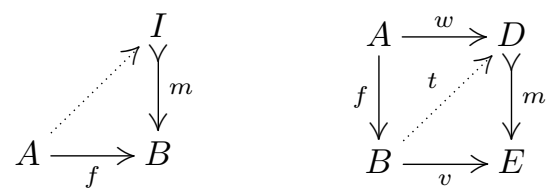

- a strong epimorphism if, for every commutative square as on the right with $m$ a monomorphism, there exists a (unique) morphism $t: B \rightarrow D$ such that $v=m \circ t($ and $t \circ f=w)$. 
It turns out that the two concepts are equivalent and that, when $\mathscr{C}$ is semi-abelian, both concepts coincide with the notions of regular and of normal epimorphism, so with the surjective homomorphisms in case $\mathscr{C}$ is a variety of algebras. However, to be a split epimorphism is a stronger condition, and to be an epimorphism is weaker.

\subsection{Cospans}

Let $\mathscr{C}$ be finitely complete. A cospan in $\mathscr{C}$ is a pair $(f, g)$

$$
A \stackrel{f}{\longrightarrow} B \leftarrow \text { g } C
$$

of morphisms in $\mathscr{C}$. We will make use of the category Cospan $(\mathscr{C})$ of cospans. A morphism $(a, b, c):\left(f^{\prime}, g^{\prime}\right) \rightarrow(f, g)$ is a commutative diagram

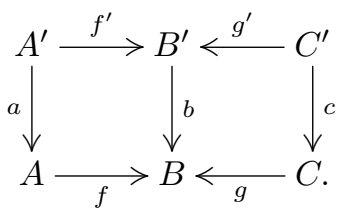

A cospan such as $(f, g)$ in $(\mathbf{B})$ is said to be

- jointly extremally epimorphic if, for every commutative diagram as on the left with $m$ a monomorphism, $m$ is an isomorphism;
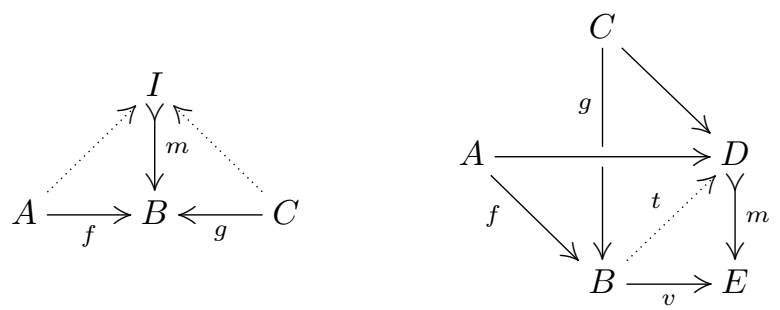

- jointly strongly epimorphic if, for every commutative diagram of solid arrows as on the right with $m$ a monomorphism, there exists a (unique) morphism $t: B \rightarrow D$ such that $v=$ mot.

As above in Section 2.3, the two concepts are actually equivalent. We may view such a cospan as a way of surjectively covering the object $B$ with the objects $A$ and $C$.

It is easily seen that a jointly strongly epimorphic pair $(f, g)$ in a regular category with binary sums is the same thing as a regular epimorphism $(f g): A+C \rightarrow B$ with a coproduct $A+C$ as domain. Consequently, in such a category, the full subcategory of Cospan $(\mathscr{C})$ determined by the jointly strongly epimorphic pairs is closed under regular quotients. There is also the following, closely related result, which follows easily from the definitions:

Lemma 2.2. In a finitely complete category, consider a commutative diagram such as $(\mathbf{C})$. If a and $c$ are strong epimorphisms and the pair $(f, g)$ is jointly strongly epimorphic, then $b$ is a strong epimorphism.

Proof. Pulling any monomorphism $m$ through which the cospan $(f \circ a, g \circ c)$ would factor back along the morphisms $f$ and $g$, then using that the morphisms $a, c$ and 
the pair $(f, g)$ are extremally epimorphic, it is easily shown that $b$ is an extremal epimorphism as well.

A projective presentation $p: P \rightarrow A$ of an object $A$ is a regular epimorphism where the object $P$ is projective (with respect to the class of regular epimorphisms). A category $\mathscr{C}$ has enough projectives when for every object a projective presentation exists. For instance, in a variety of algebras we may always take the canonical homomorphism $L U(A) \rightarrow A$, from the free algebra $L U(A)$ on the underlying set $U(A)$ of $A$, to the given algebra $A$.

Proposition 2.3. Let $\mathscr{C}$ be finitely (co)complete with enough projectives. Any pushout of split monomorphisms in $\mathscr{C}$ may be covered by a pushout in $\operatorname{Arr}(\mathscr{C})$ of split monomorphisms of presentations.

Proof. Given a pushout of split monomorphisms as on the left
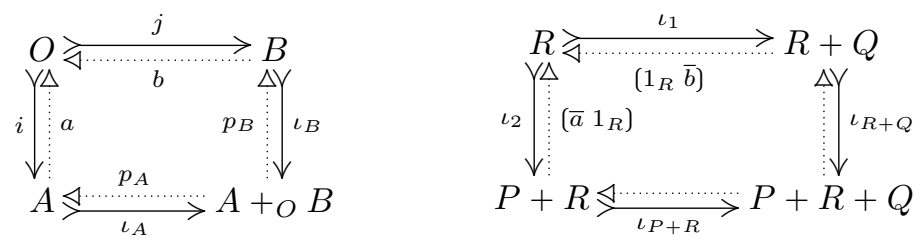

we consider projective presentations $p: P \rightarrow A, q: Q \rightarrow B$ and $r: R \rightarrow O$, and construct the pushout on the right. Note that all objects in it are projective. Since the squares of solid arrows
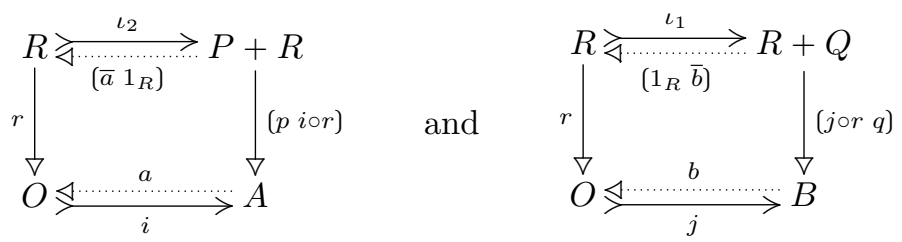

commute, Lemma 2.2 tells us that the induced arrow

$$
(p i \circ r)+{ }_{r}(j \circ r q): P+R+Q \rightarrow A+o B
$$

between the pushouts is a projective presentation.

We let $\bar{a}$ and $\bar{b}$ be liftings in the diagram

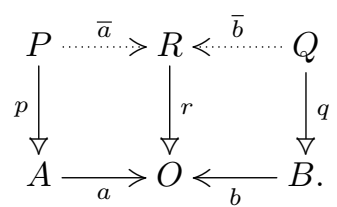

These make the two above squares commute to the left, so that the original pushout square of split monomorphisms is covered by a pushout of projectives in a way which is compatible with the given splittings. 


\subsection{Algebraically coherent categories}

A category $\mathscr{C}$ is algebraically coherent [24] when it is finitely complete and for every morphism $f: A \rightarrow B$ in $\mathscr{C}$, the change of base functor

$$
f^{*}: \mathrm{Pt}_{B}(\mathscr{C}) \rightarrow \mathrm{Pt}_{A}(\mathscr{C})
$$

is coherent, which means that it preserves finite limits and jointly strongly epimorphic pairs. In the semi-abelian context, a simple argument shows that this condition is equivalent to the coherence of the "kernel functors"

$$
\text { Ker }=!_{B}^{*}: \operatorname{Pt}_{B}(\mathscr{C}) \rightarrow \operatorname{Pt}_{0}(\mathscr{C}) \cong \mathscr{C}
$$

forgetting the $B$-action, which can be expressed quite easily as follows.

Proposition 2.4. A semi-abelian category $\mathscr{C}$ is algebraically coherent if and only if given any cospan of points over any object $B$

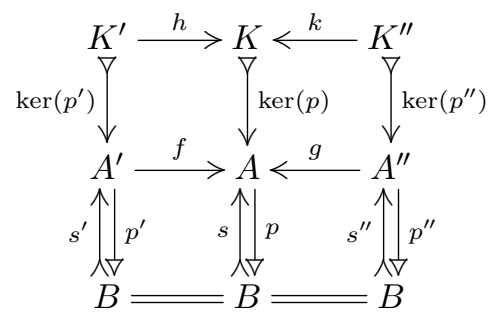

where the pair $(f, g)$ is jointly strongly epimorphic in $\mathscr{C}$, the pair $(h, k)$ is also jointly strongly epimorphic in $\mathscr{C}$.

In other words, if a $B$ acts on $K, K^{\prime}$ and $K^{\prime \prime}$, and the objects $K^{\prime}$ and $K^{\prime \prime}$ cover $K$ in the category of $B$-actions in $\mathscr{C}$, then $K^{\prime}$ and $K^{\prime \prime}$ cover $K$ in the underlying category $\mathscr{C}$.

Algebraically coherent semi-abelian categories have good stability properties. For instance, categories of diagrams and categories of regular epimorphisms in an algebraically coherent category are also algebraically coherent, as is any regular epireflective subcategory. Furthermore, as explained in [24], algebraic coherence has important categorical-algebraic consequences, such as the Smith is Huq property [57], normality of Higgins commutators [25], or strong protomodularity [12, 63].

\subsection{Examples}

Any additive category, in particular, any almost abelian category, is semi-abelian and algebraically coherent. On the other hand, not every semi-abelian category is: for instance, the categories of loops and of Jordan algebras are not. Examples of algebraically coherent semi-abelian categories are the categories of groups, associative algebras, Lie algebras, Leibniz algebras, Poisson algebras over a commutative ring with unit, all Neumann varieties of groups [59], all Orzech categories of interest [60], next to the categories of rings, crossed modules, and cocommutative Hopf algebras over a field of characteristic zero [38].

\subsection{Almost abelian categories}

A category $\mathscr{C}$ is almost abelian [66] (or Raikov semiabelian [62]) when both $\mathscr{C}$ and $\mathscr{C}^{\text {op }}$ are homological [65]. The original definition is the following: $\mathscr{C}$ is almost 
abelian if it is additive, every morphism has a kernel and a cokernel, normal epimorphisms are stable under pullbacks and normal monomorphisms are stable under pushouts. Examples of almost abelian categories are the categories of locally compact abelian groups, normed vector spaces, Banach spaces (with morphisms the bounded linear maps) and Fréchet spaces.

In such a category, every regular monomorphism (equalizer of some parallel pair of morphisms) is a normal monomorphism (a kernel).

We will use the following proposition (see [11] and [64, Lemma 1.8]):

Proposition 2.5. Let $\mathscr{C}$ be an almost abelian category and

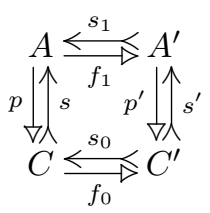

be an object of $\mathrm{Pt}^{2}(\mathscr{C})$, so that every square in $(\mathrm{D})$ commutes and

$$
f_{1} \circ s_{1}=1_{A^{\prime}}, \quad f_{0} \circ s_{0}=1_{C^{\prime}}, \quad p \circ s=1_{C}, \quad p^{\prime} \circ s^{\prime}=1_{C^{\prime}} .
$$

Then the square $f_{0} \circ p=p^{\prime} \circ f_{1}$ is a pullback if and only if $s \circ s_{0}=s_{1} \circ s^{\prime}$ is a pushout. Hence, in a diagram as above, the comparison morphism $\left\langle p, f_{1}\right\rangle: A \rightarrow C \times{ }_{C^{\prime}} A^{\prime}$ always has a section $\nu: C \times_{C^{\prime}} A^{\prime} \rightarrow A$ such that

$$
s=\nu \circ\left\langle 1_{C}, s^{\prime} \circ f_{0}\right\rangle, \quad s_{1}=\nu \circ\left\langle s_{0} \circ p^{\prime}, 1_{A^{\prime}}\right\rangle .
$$

Then the square $f_{0} \circ p=p^{\prime} \circ f_{1}$ is a pullback precisely when the pair $\left(s, s_{1}\right)$ is jointly epimorphic.

\section{Fundamental group functors and derived functors}

Our proof of the Seifert-van Kampen theorem is based on an interpretation of the first left derived functor $\mathscr{L}_{1} F$ of a given functor $F: \mathscr{C} \rightarrow \mathscr{X}$ as a fundamental group functor relative to $F$, defined via categorical Galois theory. Thus the context where our result applies is, first of all, limited by the scope of the latter general framework. We consider a fixed adjunction

$$
\mathscr{C} \underset{\frac{H}{H}}{\frac{1}{<}} \mathscr{X}
$$

with unit $\eta: 1_{\mathscr{C}} \Rightarrow H \circ F$ and counit $\epsilon: F \circ H \Rightarrow 1_{\mathscr{X}}$ such that

- $\mathscr{C}$ is semi-abelian and algebraically coherent with enough projectives;

- $\mathscr{X}$ is almost-abelian;

- $\eta_{C}: C \rightarrow H F(C)$ is a regular epimorphism for all $C$ in $\mathscr{C}$;

- $\epsilon_{X}: F H(X) \rightarrow X$ is an isomorphism for all $X$ in $\mathscr{X}$;

- $F$ preserves pullbacks of split epimorphisms along regular epimorphisms.

Such an adjunction naturally gives rise to a closed Galois structure $\Gamma$; in the sense of [47]. We call the regular epimorphisms in $\mathscr{C}$ extensions and we denote by 
$\operatorname{Ext}(\mathscr{C})$ the full subcategory of the category $\operatorname{Arr}(\mathscr{C})$ of arrows in $\mathscr{C}$ determined by the extensions; morphisms are commutative squares between them. Since the condition on $\epsilon$ makes $\mathscr{X}$ a reflective subcategory of $\mathscr{C}$, we shall sometimes omit the right adjoint inclusion $H$. It is well known that the condition on the $\eta_{C}$ being regular epimorphisms is equivalent to $\mathscr{X}$ being closed under subobjects in $\mathscr{C}$.

\subsection{Trivial and normal extensions}

With respect to a closed Galois structure $\Gamma$ as above, an extension $f: A \rightarrow B$ is said to be trivial if the naturality square

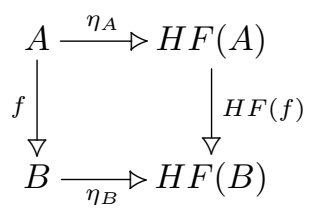

is a pullback, and normal if the first projection $\operatorname{pr}_{1}^{f}=f^{*}(f): \operatorname{Eq}(f) \rightarrow A$ of the kernel pair of $f$ is trivial. We write $\operatorname{NExt}(\mathscr{C})$ for the category of normal extensions, considered as a full subcategory of $\operatorname{Ext}(\mathscr{C})$. Any trivial extension is a normal extension, and any split epimorphism which is a normal extension is automatically a trivial extension (see [29, Lemma 11] or [16, Corollary 2.11]). Normal extensions should not be confused with normal epimorphisms (cokernels), since by definition extensions and normal epimorphisms are precisely the same thing, whereas normal extensions are introduced in order to model concepts such as central extensions of groups.

\subsection{Normalization}

The inclusion functor $H_{1}: \operatorname{NExt}(\mathscr{C}) \rightarrow \operatorname{Ext}(\mathscr{C})$ has a left adjoint $F_{1}$, called the normalization functor. The component of the unit at an extension $f: A \rightarrow B$ is of the form $\left(\rho_{f}^{1}, 1_{B}\right)$

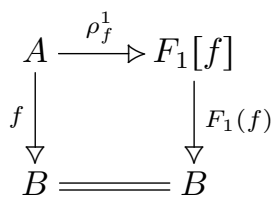

where $\rho_{f}^{1}$ is a regular epimorphism. Furthermore, $F\left(\rho_{f}^{1}\right)$ is an isomorphism. As a consequence:

Proposition 3.1. The full subcategory $\operatorname{NExt}(\mathscr{C})$ determined by the normal extensions is a regular epi-reflective subcategory of the category $\operatorname{Ext}(\mathscr{C})$.

See [28] for a more complete account. This allows us to prove the following lemma, needed in Section 4.

Lemma 3.2. If $n: N \rightarrow K$ is a split monomorphism and $k: K \rightarrow A$ is the kernel of a trivial extension, then the composite monomorphism $k \circ n: N \rightarrow A$ is also the kernel of a trivial extension. 
Proof. Let $f: A \rightarrow B$ be a trivial extension of which $k$ is the kernel, and let us consider the commutative diagram

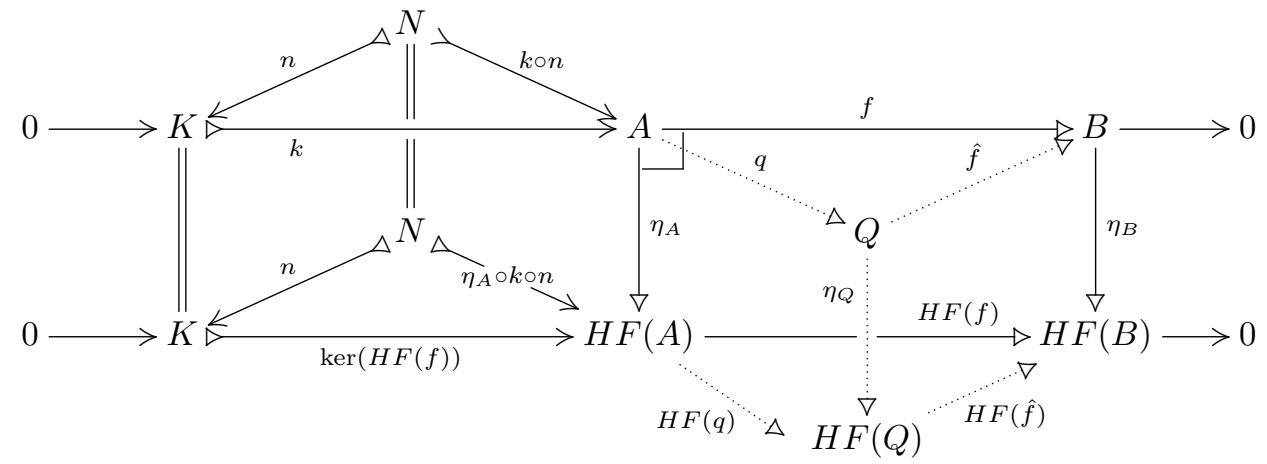

where $q$ is the cokernel of the composite $k \circ n$. Since $f$ is a trivial extension, the bottom right square is a pullback, which implies that the kernel of $H F(f)$ is the composite $\eta_{A^{\circ}} k: K \rightarrow H F(A)$. In particular, the object $K$ lies in $\mathscr{X}$. Since $\mathscr{X}$ is closed under subobjects in $\mathscr{C}$, also $N$ is in $\mathscr{X}$. Hence $\eta_{N}=1_{N}$, so that $F(k \circ n)=$ $\eta_{A^{\circ}} \circ \circ n$. This latter composite $\eta_{A} \circ k \circ n$ is a normal monomorphism as a composite of two regular monomorphisms $(\operatorname{ker}(H F(f))$ and $n)$ in the cohomological category $\mathscr{X}$. Since the left adjoint $F$ preserves cokernels,

$$
F(q)=\operatorname{coker}(F(k \circ n))=\operatorname{coker}\left(\eta_{A} \circ k \circ n\right) .
$$

As a consequence, $\eta_{A} \circ k \circ n$ is the kernel of $H F(q)$. It follows that $k \circ n$ is the kernel of $q$, since $q$ is a trivial extension, so that the square $\eta_{Q^{\circ} q}=H F(q) \circ \eta_{A}$ is a pullback.

This latter claim still remains to be shown. On the one hand, it is easily verified by hand that the square $\eta_{Q^{\circ}} q=H F(q) \circ \eta_{A}$ is a pushout. Hence Lemma 2.1 tells us that the comparison morphism $\left\langle\eta_{A}, q\right\rangle: A \rightarrow H F(A) \times_{H F(Q)} Q$ is a regular epimorphism. On the other hand, since the square $\eta_{B} \circ=H F(f) \circ \eta_{A}$ is a pullback, the pair $\left(\eta_{A}, f\right)$ is jointly monomorphic. Hence also $\eta_{A}$ and $q$ are jointly monomorphic, so that $\left\langle\eta_{A}, q\right\rangle$ is an isomorphism, and the claim follows.

\subsection{First fundamental group functor and Hopf formula}

As explained in the introduction, the first fundamental group functor is defined as a pointwise right Kan extension: $\pi_{1}^{F}=\operatorname{Ran}_{\mathrm{Cod}}\left(\operatorname{Ker} \circ F_{1}\right)$,

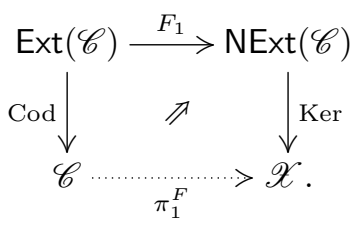

Its value in some object $A$ can be described by a so-called Hopf formula-first obtained in $[43,18,34,27,33,30]$, then extended to the present context in [28].

Given a projective presentation $p: P \rightarrow A$ of $A$, we have

$$
\pi_{1}^{F}(A) \cong \frac{\operatorname{Ker}(p) \cap \operatorname{Ker}\left(\eta_{P}\right)}{\operatorname{Ker}\left(\rho_{p}^{1}\right)} .
$$


Using this, we easily find that

$$
\pi_{1}^{F}(A) \cong \operatorname{Ker}(u) \cap \operatorname{Ker}\left(\eta_{U}\right)
$$

for $u:=F_{1}(p): U \rightarrow A$, so that there is an exact sequence

$$
0 \longrightarrow \pi_{1}^{F}(A) \longmapsto \operatorname{Ker}(u) \longrightarrow H F(U) .
$$

Actually, there exist morphisms of short exact sequences

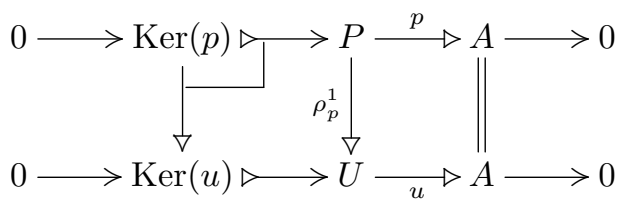

and

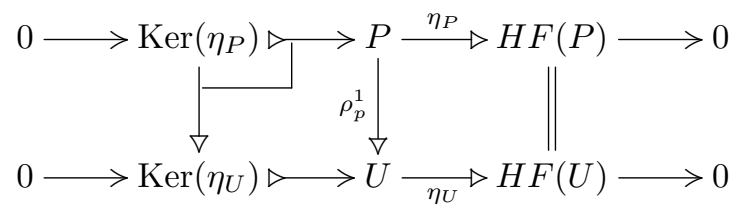

so that by using some standard compositions / cancelations of pullbacks, we may see that $(\mathbf{F})$ holds.

\subsection{Birkhoff subcategories}

When the subcategory $\mathscr{X}$ is closed under regular quotients, so that $\mathscr{X}$ is a Birkhoff subcategory [49] of $\mathscr{C}$, results of [30] show that the first fundamental group functor is the left derived functor of the reflector $F$ : we have an isomorphism $\pi_{1}^{F} \cong \mathscr{L}_{1}(F)$. An important class of examples occurs when $F$ is the reflector from a semi-abelian algebraically coherent variety $\mathscr{C}$ to an abelian subvariety $\mathscr{X}$ of $\mathscr{C}$. For instance, the reflector from groups to abelian groups, from groups of a certain nilpotency class to abelian groups, from crossed modules to abelian crossed modules, from Lie algebras over a field $\mathbb{K}$ to $\mathbb{K}$-vector spaces, from $G$-actions to $\mathbb{Z}[G]$-modules for a given group $G$.

By Corollary 9 and Example 13 in [8] combined, the condition that $\mathscr{X}$ is a Birkhoff subcategory of $\mathscr{C}$ is equivalent to its exactness. That is to say, $\mathscr{X}$ is abelian (= additive + exact, by Tierney's result [1]) if and only if it is Birkhoff. Note, furthermore, that any Birkhoff reflector preserves pullbacks of regular epimorphisms along split epimorphisms [37, 33].

Other (non-Birkhoff) examples are, for instance, the reflection of Gp to the category of torsion-free abelian groups, or the "reflection" from the category of cocommutative Hopf algebras over a field of characteristic zero to the category of abelian groups (considered as group Hopf algebras).

\section{Some results on jointly strongly epimorphic pairs}

Here we prove some technical results having to do with the preservation of jointly strongly epimorphic pairs by kernel functors in algebraically coherent categories. We 
start with Proposition 4.3, a variation on Proposition 2.4.

Lemma 4.1 ([25, Lemma 2.6]). In a semi-abelian category, consider a point with chosen kernel as in the bottom row of the diagram

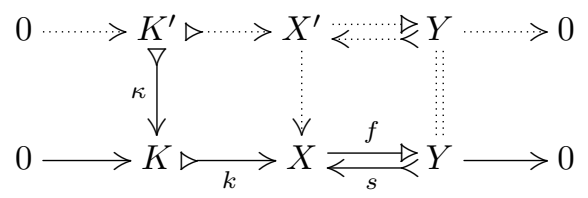

and a monomorphism $\kappa: K^{\prime} \rightarrow K$ such that $k \circ \kappa$ is a normal monomorphism. Then this point lifts along $\kappa$ to yield a morphism of points with chosen kernels. Moreover, this process is functorial in the data given.

The functoriality in the above lemma implies the following.

Lemma 4.2. In a semi-abelian category, given a morphism of split extensions such as

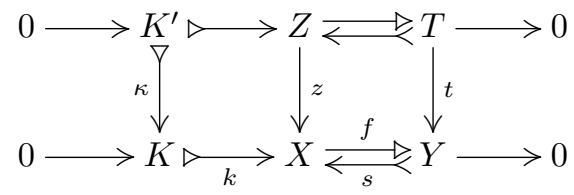

where $k \circ \kappa$ is a normal monomorphism, this morphism factors as a composite

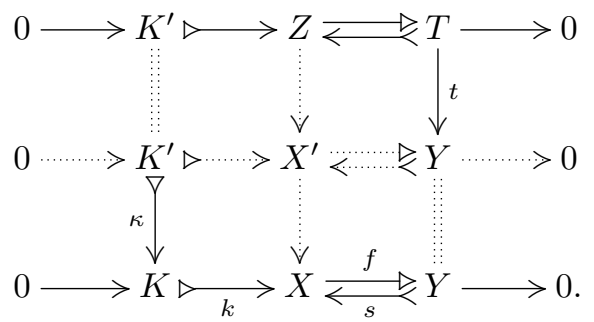

Proof. The needed factorization is induced by Lemma 4.1 as in the square of split extensions of Figure 1.

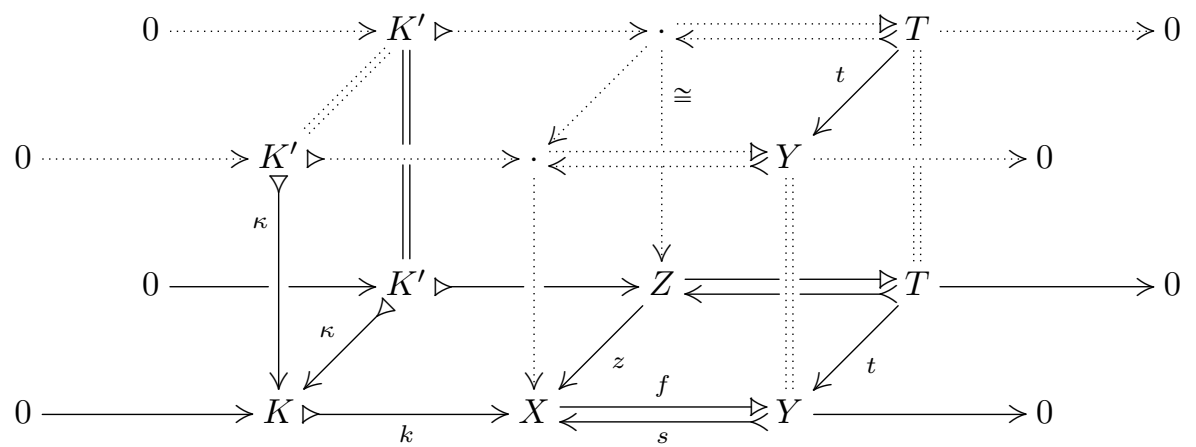

Figure 1: A square of split extensions induced by Lemma 4.1. 
Proposition 4.3. In an algebraically coherent semi-abelian category, consider a cospan of split extensions

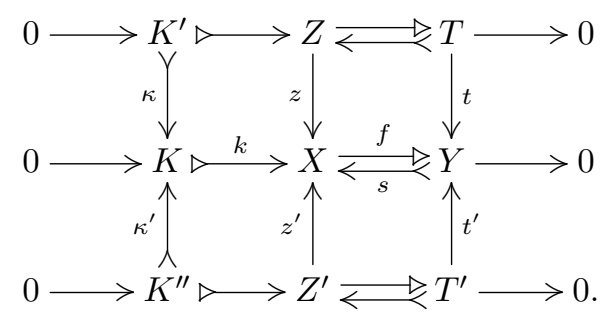

If $f$ is a trivial extension, $\kappa$ and $\kappa^{\prime}$ are split monomorphisms and $\left(z, z^{\prime}\right)$ is a jointly strongly epimorphic pair, then also the pair $\left(\kappa, \kappa^{\prime}\right)$ is jointly strongly epimorphic.

Proof. Lemma 3.2 tells us that the composites $k \circ \kappa$ and $k \circ \kappa^{\prime}$ are normal monomorphisms. Hence we can apply Lemma 4.2 twice to reduce the situation to the special case where $t$ and $t^{\prime}$ are identities. The result then follows from algebraic coherence via Proposition 2.4.

When the cospans under consideration are induced by squares of split monomorphisms, this result may be refined as in Proposition 4.5.

We say that a commutative square

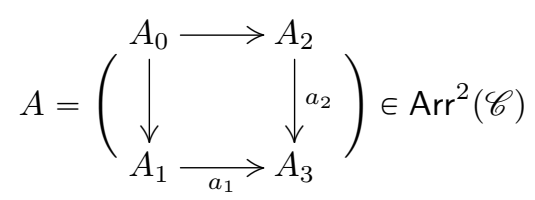

is jointly strongly epimorphic when such is its underlying cospan $\left(a_{1}, a_{2}\right)$. Note that a pushout square is always jointly strongly epimorphic.

Lemma 4.4. Suppose that $\mathscr{C}$ is semi-abelian. Consider in $\operatorname{Arr}^{2}(\mathscr{C})$ a pullback of regular epimorphisms

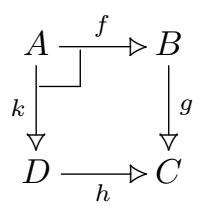

where $B$ and $D$ are jointly strongly epimorphic squares and $C$ is a pushout square. Then $A$ is also a jointly strongly epimorphic square.

Proof. Let us consider the commutative diagram of solid arrows in Figure 2, where the objects and arrows in $\mathscr{C}$ are numbered as in $(\mathbf{H})$. Lemma 2.2 says that both $\bar{k}_{3}:=k_{3} \circ\left(a_{1} a_{2}\right): A_{1}+A_{0} A_{2} \rightarrow D_{3}$ and $\bar{f}_{3}:=f_{3} \circ\left(a_{1} a_{2}\right): A_{1}+A_{A_{0}} A_{2} \rightarrow B_{3}$ are regular epimorphisms. By Lemma 2.1, it remains to show that the square $g_{3} \circ \bar{f}_{3}=h_{3} \circ \bar{k}_{3}$ is a pushout. This is easily checked using the definition of pushouts and the fact that $C$ is a pushout square. 


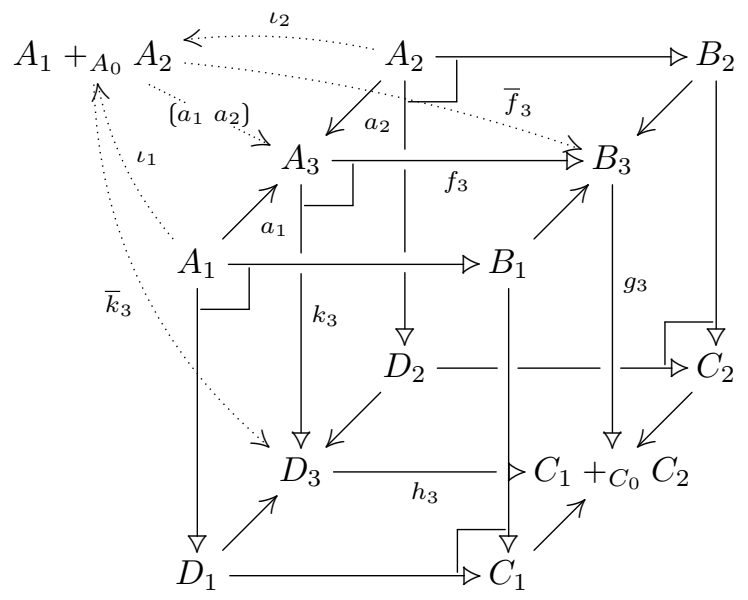

Figure 2: Situation sketch for Lemma 4.4.

Proposition 4.5. Let

$$
0 \longrightarrow K \triangleright^{k} \longrightarrow A \stackrel{f}{\longrightarrow} B \longrightarrow 0
$$

be a short exact sequence in $\mathrm{Pt}^{2}(\mathscr{C})$ where $f$ is component-wise a normal extension. If $A$, considered as a square of split monomorphisms, is jointly strongly epimorphic, and $B$ is a pushout square, then $K$ is also a jointly strongly epimorphic square.

Proof. Lemma 4.4 allows us to reduce the situation to the special case where $f$ is a both a split epimorphism and a trivial extension. Indeed, the pullback $f^{*}(f)$ of $f$ along itself is a split epimorphic normal extension, which makes it a trivial extension - see Section 3.1. Furthermore, the kernel of $f^{*}(f)$ is $K$. The conclusion now follows from Lemma 4.3.

\section{The Seifert-van Kampen theorem}

We are now ready to prove our main result. We start with a version of the Seifertvan Kampen theorem for fundamental group functors, Theorem 5.1. We then restrict our attention to the situation where those fundamental group functors are the derived functors of a Birkhoff reflector and obtain Theorem 5.2. After that we finish with some final questions and comments.

Theorem 5.1. Let $\Gamma$ be a Galois structure as in $(\mathbf{E})$ and let us consider in the category $\mathscr{C}$ a pushout of split monomorphisms as on the left.
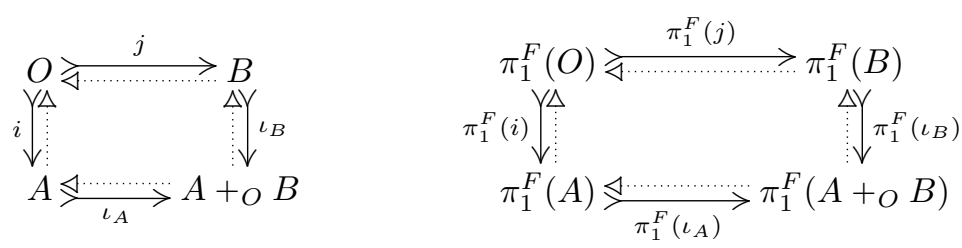
Then the square on the right is a pushout square in $\mathscr{X}$.

Proof. Proposition 2.3 gives us a way of covering the given pushout square by a pushout of projective presentations as in the left hand side cube of Figure 3. Normal-
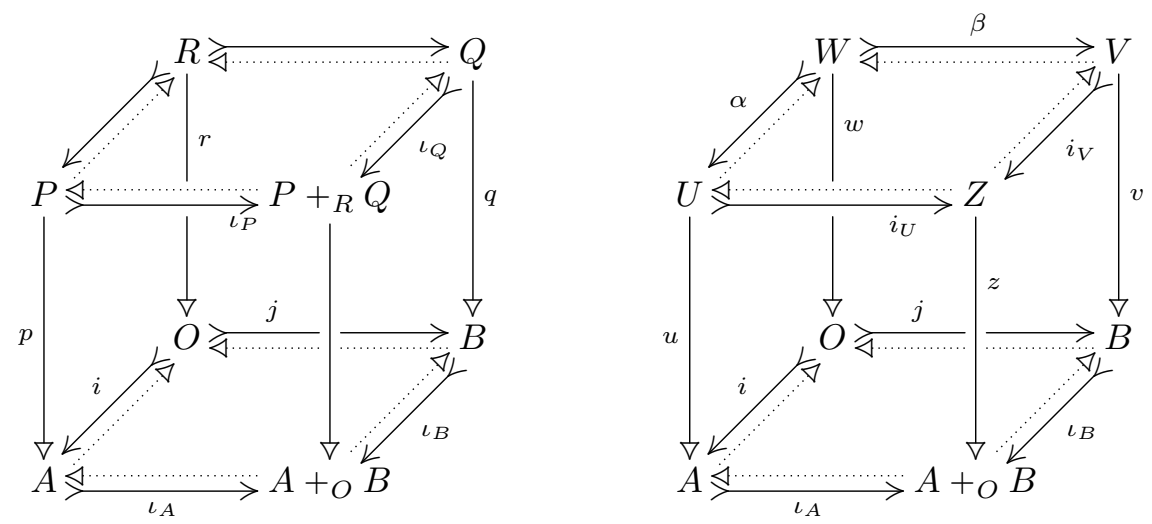

Figure 3: A projective cover of a pushout square, and its normalization.

izing, we obtain a square in $\operatorname{NExt}(\mathscr{C})$ represented by the right hand side cube. The top diagram in this cube is an object of $\operatorname{Pt}^{2}(\mathscr{C})$, in which the solid square is jointly strongly epimorphic: $\left(i_{U}, i_{V}\right)$ is a jointly strongly epimorphic pair, as a regular quotient of the pair $\left(\iota_{P}, \iota_{Q}\right)$.

In Figure 4, all rows are exact as in $(\mathbf{G})$. In order to prove our claim, we only need

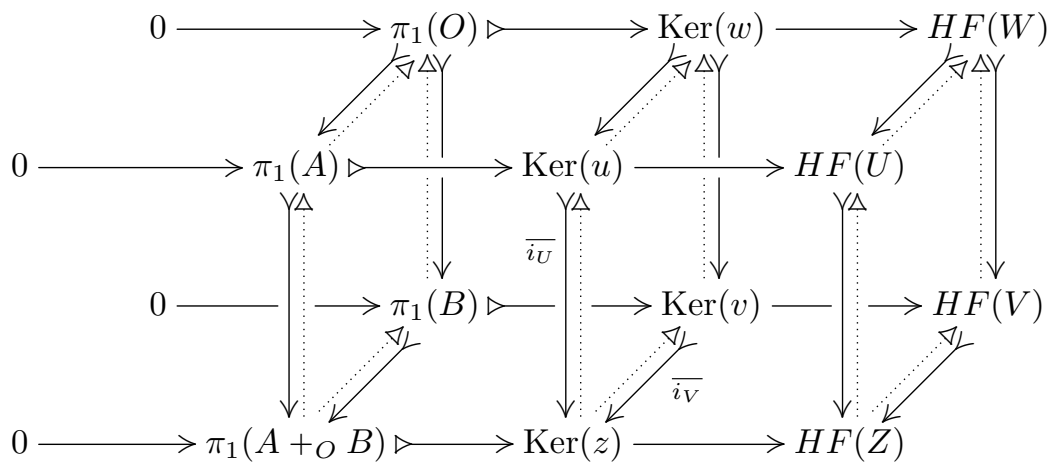

Figure 4: The exact sequence $(\mathbf{G})$ induced by the right hand side cube of normal extensions in Figure 3.

to show that the central and the right hand side upward directed dotted squares of this figure are pullbacks. Indeed, then the dotted square on the left is a pullback as well, and the result follows from Proposition 2.5.

We first show that the right hand side dotted square in Figure 4 is a pullback. Recalling from Subsection 3.2 that $F(P) \cong F(U), F(Q) \cong F(V), F(R) \cong F(W)$ and 
$F\left(P+{ }_{R} Q\right) \cong F(Z)$, we see that the two cubes in Figure 3 have the same image through $F$. Since the reflector $F$ preserves colimits, we find that the solid square in

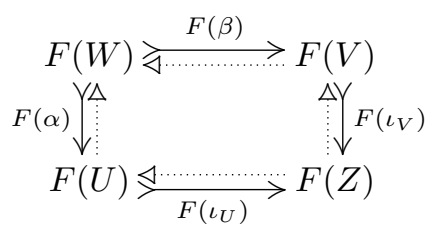

is a pushout diagram in $\mathscr{X}$. Consequently, by Proposition 2.5 the dotted square in the same diagram is a pullback, which is preserved by the right adjoint inclusion $H$.

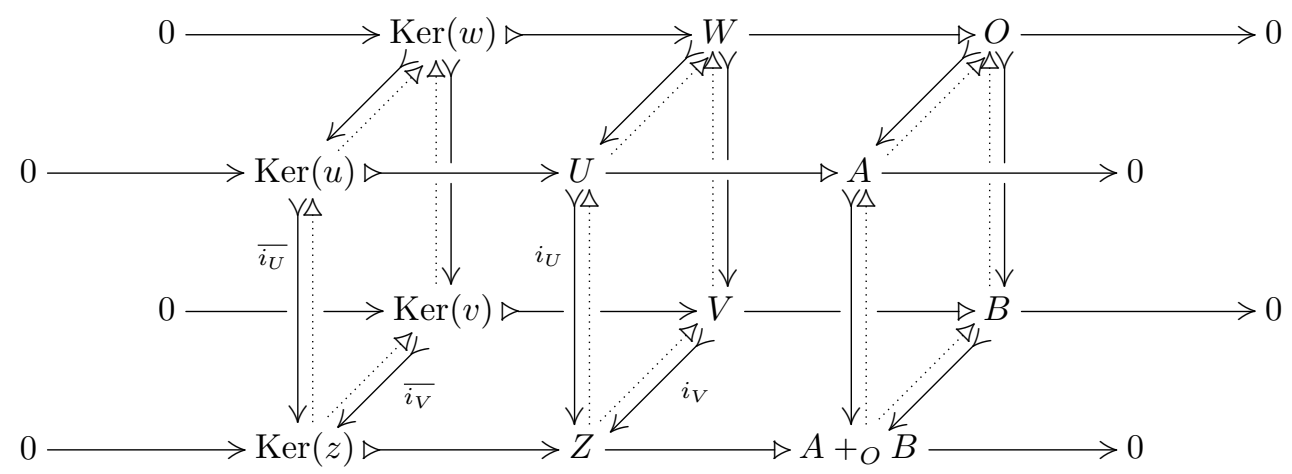

Figure 5: Taking kernels in the right hand side cube of Figure 3 yields a short exact sequence in $\operatorname{Pt}^{2}(\mathscr{C})$.

Let us now prove that also the central dotted square in Figure 4 is a pullback. Again by Proposition 2.5, for this it suffices that the pair $\left(\overline{i_{U}}, \overline{i_{V}}\right)$ is jointly (strongly) epimorphic in $\mathscr{X}$ or, equivalently, in $\mathscr{C}$. To see this, we consider the diagram in Figure 5 and apply Proposition 4.5.

Theorem 5.2. Let $F: \mathscr{C} \rightarrow \mathscr{X}$ be the Birkhoff reflector from a semi-abelian algebraically coherent category with enough projectives $\mathscr{C}$ to an abelian category $\mathscr{X}$. The functor $F$ may, for instance, be the reflector from an Orzech category of interest to an abelian subvariety. Consider in $\mathscr{C}$ a pushout of split monomorphisms as on the left.
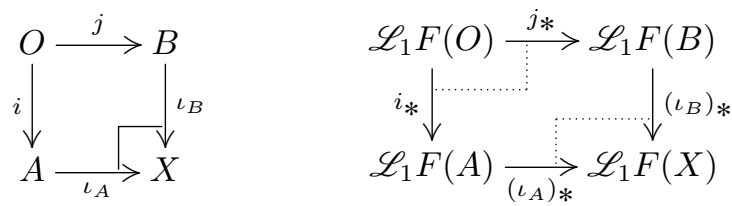

Then the square on the right is a pushout square in $\mathscr{X}$. Moreover, we have "MayerVietoris" short exact sequences $[58,73]$

$$
0 \longrightarrow \mathscr{L}_{n} F(O) \stackrel{\left\langle i_{*}, j_{*}\right\rangle}{\longrightarrow} \mathscr{L}_{n} F(A) \oplus \mathscr{L}_{n} F(B) \stackrel{\left(\left(\iota_{A}\right)_{*}-\left(\iota_{B}\right)_{*}\right)}{\longrightarrow} \mathscr{L}_{n} F(X) \longrightarrow 0
$$

for $n \in\{0,1\}$. As a consequence, the pushout on the right is also a pullback. 
Proof. The pushout result is Theorem 5.1 via the interpretation of left derived functors as Galois groups, taking the discussion in Subsection 3.4 into account. Recall that $\mathscr{L}_{0} F=F$ preserves pushouts since it is a left adjoint functor. The short exact sequences follow from the construction of pushouts in abelian categories [35, Proposition 2.53], and the fact that the composite

$$
\mathscr{L}_{n} F(O) \stackrel{\Delta_{\mathscr{L}_{n} F(O)}}{\longrightarrow} \mathscr{L}_{n} F(O) \oplus \mathscr{L}_{n} F(O) \stackrel{i_{*} \oplus j_{*}}{\longrightarrow} \mathscr{L}_{n} F(A) \oplus \mathscr{L}_{n} F(B)
$$

is a (split) monomorphism.

Remark 5.3. One technique used in [3] by Barr and Beck in order to obtain a homology coproduct theorem such as (A) is to assume that a sum of two resolutions is again a resolution. This hardly ever happens, so this technique is of limited use when trying to prove general results. However, a trace of their idea occurs in the proof of Theorem 5.1, when Proposition 2.3 is used to obtain a presentation of a pushout square.

Following the interpretation in [31] of $n$-fold extensions as finite-dimensional resolutions, part of Barr and Beck's argument may be recoverable when the sum of two $n$-fold extensions is still an $n$-fold extension. We do not know how this condition relates to other conditions studied in categorical algebra.

Remark 5.4. Another categorical-algebraic condition which may be related to the problem of extending Theorem 5.2 to higher degrees is the following. In $[39,14]$ a semi-abelian variety is called locally algebraically cartesian closed (LACC) when the change of base functors considered in Section 2.5 preserve binary coproducts. It is easy to see that this condition implies algebraic coherence. Examples include the categories of groups, Lie algebras, crossed modules, cocommutative Hopf algebras over a field of characteristic zero. On the other hand, the category $\mathrm{Nil}_{2}$ of groups of nilpotency class at most 2 is not an example. Incidentally, $\mathrm{Nil}_{2}$ is the counterexample from [51] which appeared on page 81. Since (LACC) is one of the rare categoricalalgebraic conditions satisfied by $\mathrm{Gp}$ but not by $\mathrm{Nil}_{2}$, perhaps a higher-order version of Theorem 5.1 is valid for (LACC) semi-abelian categories with enough projectives.

\section{References}

[1] M. Barr, Exact categories, Exact Categories and Categories of Sheaves, Lecture Notes in Math., vol. 236, Springer, 1971, pp. 1-120.

[2] M. Barr and J. Beck, Acyclic models and triples, Proceedings of the Conference on Categorical Algebra, La Jolla, 1965 (S. Eilenberg, D.K. Harrison, S. Mac Lane, and H. Röhrl, eds.), Springer, 1966, pp. 336-343.

[3] M. Barr and J. Beck, Homology and standard constructions, Seminar on Triples and Categorical Homology Theory (ETH, Zürich, 1966/67), Lecture Notes in Math., vol. 80, Springer, 1969, pp. 245-335.

[4] J.M. Beck, Triples, algebras and cohomology, Repr. Theory Appl. Categ. 2 (2003), 1-59, Ph.D. thesis, Columbia University, 1967.

[5] I. Bernstein, On co-groups in the category of graded algebras, Trans. Amer. Math. Soc. 115 (1965), 257-269. 
[6] F. Borceux, Handbook of Categorical Algebra 2: Categories and Structures, Encyclopedia Math. Appl., vol. 51, Cambridge Univ. Press, 1994.

[7] F. Borceux and D. Bourn, Mal'cev, Protomodular, Homological and SemiAbelian Categories, Math. Appl., vol. 566, Kluwer Acad. Publ., 2004.

[8] F. Borceux, M.M. Clementino, M. Gran, and L. Sousa, Protolocalisations of homological categories, J. Pure Appl. Algebra 212 (2008), 636-651.

[9] F. Borceux and G. Janelidze, Galois Theories, Cambridge Stud. Adv. Math., vol. 72, Cambridge Univ. Press, 2001.

[10] D. Bourn, Normalization equivalence, kernel equivalence and affine categories, Category Theory, Proceedings Como 1990 (A. Carboni, M.C. Pedicchio, and G. Rosolini, eds.), Lecture Notes in Math., vol. 1488, Springer, 1991, pp. 43-62.

[11] D. Bourn, Mal'cev categories and fibration of pointed objects, Appl. Categ. Structures 4 (1996), 307-327.

[12] D. Bourn, Normal functors and strong protomodularity, Theory Appl. Categ. 7 (2000), no. 9, 206-218.

[13] D. Bourn, Protomodular aspect of the dual of a topos, Adv. Math. 187 (2004), 240-255.

[14] D. Bourn and J.R.A. Gray, Aspects of algebraic exponentiation, Bull. Belg. Math. Soc. Simon Stevin 19 (2012), 823-846.

[15] D. Bourn and G. Janelidze, Protomodularity, descent, and semidirect products, Theory Appl. Categ. 4 (1998), no. 2, 37-46.

[16] D. Bourn and D. Rodelo, Comprehensive factorization and I-central extensions, J. Pure Appl. Algebra 216 (2012), 598-617.

[17] R. Brown, Groupoids and Van Kampen's theorem, Proc. Lond. Math. Soc. 17 (1967), 385-401.

[18] R. Brown and G.J. Ellis, Hopf formulae for the higher homology of a group, Bull. Lond. Math. Soc. 20 (1988), no. 2, 124-128.

[19] R. Brown and G. Janelidze, Van Kampen theorems for categories of covering morphisms in lextensive categories, J. Pure Appl. Algebra 119 (1997), 255-263.

[20] R. Brown and J.-L. Loday, Van Kampen theorems for diagrams of spaces, Topology 26 (1987), no. 3, 311-335.

[21] M. Bunge and S. Lack, van Kampen theorems for toposes, Adv. Math. 179 (2003), 291-317.

[22] A. Carboni, G.M. Kelly, and M.C. Pedicchio, Some remarks on Maltsev and Goursat categories, Appl. Categ. Structures 1 (1993), 385-421.

[23] H. Cartan and S. Eilenberg, Homological Algebra, Princeton University Press, 1956.

[24] A.S. Cigoli, J.R.A. Gray, and T. Van der Linden, Algebraically coherent categories, Theory Appl. Categ. 30 (2015), no. 54, 1864-1905.

[25] A.S. Cigoli, J.R.A. Gray, and T. Van der Linden, On the normality of Higgins commutators, J. Pure Appl. Algebra 219 (2015), 897-912.

[26] A. Clark and L. Smith, The rational homotopy of a wedge, Pacific J. Math. 24 (1968), no. 2, 241-246. 
[27] G. Donadze, N. Inassaridze, and T. Porter, $n$-Fold Čech derived functors and generalised Hopf type formulas, K-Theory 35 (2005), nos. 3-4, 341-373.

[28] M. Duckerts-Antoine, Fundamental group functors in descent-exact homological categories, Adv. Math. 310 (2017), 56-120.

[29] M. Duckerts-Antoine and T. Everaert, A classification theorem for normal extensions, Preprint arXiv:1604.02604, 2016.

[30] T. Everaert, Higher central extensions and Hopf formulae, J. Algebra $\mathbf{3 2 4}$ (2010), 1771-1789.

[31] T. Everaert, J. Goedecke, and T. Van der Linden, Resolutions, higher extensions and the relative Mal'tsev axiom, J. Algebra 371 (2012), 132-155.

[32] T. Everaert, J. Goedecke, and T. Van der Linden, The fundamental group functor as a Kan extension, Cah. Topol. Géom. Differ. Catég. LIV (2013), no. $3,185-210$.

[33] T. Everaert, M. Gran, and T. Van der Linden, Higher Hopf formulae for homology via Galois Theory, Adv. Math. 217 (2008), no. 5, 2231-2267.

[34] T. Everaert and T. Van der Linden, Baer invariants in semi-abelian categories II: Homology, Theory Appl. Categ. 12 (2004), no. 4, 195-224.

[35] P. Freyd, Abelian Categories, Harper and Row, New York, 1964, Republished in: Repr. Theory Appl. Categ., no. 3 (2003).

[36] J. Goedecke and T. Van der Linden, On satellites in semi-abelian categories: Homology without projectives, Math. Proc. Cambridge Philos. Soc. 147 (2009), no. 3, 629-657.

[37] M. Gran, Applications of categorical Galois theory in universal algebra, Galois Theory, Hopf Algebras, and Semiabelian Categories, Fields Inst. Commun., vol. 43, Amer. Math. Soc., 2004, pp. 243-280.

[38] M. Gran, G. Kadjo, and J. Vercruysse, A torsion theory in the category of cocommutative Hopf algebras, Appl. Categ. Structures 24 (2016), no. 3, 269282 .

[39] J.R.A. Gray, Algebraic exponentiation in general categories, Appl. Categ. Structures 20 (2012), 543-567.

[40] A. Grothendieck and M. Raynaud, Revêtements étales et groupe fondamental (SGA1), Lecture Notes in Math., vol. 224, Springer, 1971.

[41] P.J. Higgins, Groups with multiple operators, Proc. Lond. Math. Soc. (3) 6 (1956), no. 3, 366-416.

[42] P.J. Hilton and B. Steer, On fibred categories and cohomology, Topology 8 (1969), 243-251.

[43] H. Hopf, Fundamentalgruppe und zweite Bettische Gruppe, Comment. Math. Helv. 14 (1942), 257-309.

[44] T.W. Hungerford, The free product of algebras, Illinois J. Math. 12 (1968), 312-324.

[45] S.A. Huq, Commutator, nilpotency and solvability in categories, Q. J. Math. 19 (1968), no. 2, 363-389. 
[46] G. Janelidze, Pure Galois theory in categories, J. Algebra 132 (1990), no. 2, 270-286.

[47] G. Janelidze, Categorical Galois theory: revision and some recent developments, Galois Connections and Applications, Math. Appl., vol. 565, Kluwer Acad. Publ., 2004, pp. 139-171.

[48] G. Janelidze, Galois groups, abstract commutators and Hopf formula, Appl. Categ. Structures 16 (2008), 653-668.

[49] G. Janelidze and G.M. Kelly, Galois theory and a general notion of central extension, J. Pure Appl. Algebra 97 (1994), no. 2, 135-161.

[50] G. Janelidze, L. Márki, and W. Tholen, Semi-abelian categories, J. Pure Appl. Algebra 168 (2002), no. 2-3, 367-386.

[51] K.W. Johnson, A computer calculation of homology in varieties of groups, J. Lond. Math. Soc. (2) 8 (1974), 247-252.

[52] P.T. Johnstone, A note on the semiabelian variety of Heyting semilattices, Galois Theory, Hopf Algebras, and Semiabelian Categories, Fields Inst. Commun., vol. 43, Amer. Math. Soc., 2004, pp. 317-318.

[53] C.R. Leedham-Green, Homology in varieties of groups, I, Trans. Amer. Math. Soc. 162 (1970), 1-14.

[54] S. Mac Lane, Homology, Grundlehren Math. Wiss., vol. 114, Springer, 1967.

[55] S. MacLane, Categories for the Working Mathematician, second ed., Grad. Texts in Math., vol. 5, Springer, 1998.

[56] A. Magid, A Van Kampen theorem for separable algebras, Proc. Amer. Math. Soc. 39 (1973), no. 3, 493-488.

[57] N. Martins-Ferreira and T. Van der Linden, A note on the "Smith is Huq" condition, Appl. Categ. Structures 20 (2012), no. 2, 175-187.

[58] W. Mayer, Über abstrakte Topologie, Monatsh. Math. 36 (1929), no. 1, 1-42.

[59] H. Neumann, Varieties of Groups, Ergeb. Math. Grenzgeb. (3), vol. 37, Springer-Verlag, 1967.

[60] G. Orzech, Obstruction theory in algebraic categories I and II, J. Pure Appl. Algebra 2 (1972), 287-314 and 315-340.

[61] D.G. Quillen, Homotopical Algebra, Lecture Notes in Math., vol. 43, Springer, 1967.

[62] D.A. Raïkov, Semi-abelian categories, Dokl. Akad. Nauk SSSR 188 (1969), no. 5, 1006-1009.

[63] D. Rodelo, Moore categories, Theory Appl. Categ. 12 (2004), no. 6, 237-247.

[64] D. Rodelo and T. Van der Linden, Higher central extensions and cohomology, Adv. Math. 287 (2016), 31-108.

[65] J. Rosický and W. Tholen, Factorisation, fibration and torsion, J. Homotopy Relat. Struct. 2 (2007), no. 2, 295-314.

[66] W. Rump, Almost abelian categories, Cah. Topol. Géom. Différ. Catég. XLII (2001), 163-225. 
[67] H. Seifert, Konstruction drei dimensionaler geschlossener Raume, Berichte Sachs. Akad. Leipzig, Math.-Phys. Kl. 83 (1931), 26-66.

[68] P. Stevenhagen, Unramified class field theory for orders, Trans. Amer. Math. Soc. 311 (1989), 483-500.

[69] J. Stix, A general Seifert-van Kampen theorem for algebraic fundamental groups, Publ. RIMS, Kyoto Univ. 42 (2006), 763-786.

[70] M. Tierney and W. Vogel, Simplicial derived functors, Category Theory, Homology Theory and Their Applications I, Lecture Notes in Math., vol. 86, Springer, 1969, pp. 167-180.

[71] T. Van der Linden, Simplicial homotopy in semi-abelian categories, J. K-Theory 4 (2009), no. 2, 379-390.

[72] E.R. Van Kampen, On the connection between the fundamental groups of some related spaces, Amer. J. Math. 55 (1933), no. 1, 261-267.

[73] L. Vietoris, Über die Homologiegruppen der Vereinigung zweier Komplexe, Monatsh. Math. 37 (1930), no. 1, 59-62.

[74] C.A. Weibel, An Introduction to Homological Algebra, Cambridge Stud. Adv. Math., vol. 38, Cambridge Univ. Press, 1994.

[75] V. Zoonekynd, Théorème de Van Kampen pour les champs algébriques, Ann. Math. Blaise Pascal 9 (2002), no. 1, 101-145.

Mathieu Duckerts-Antoine mathieud@mat.uc.pt

Universidade de Coimbra, Departamento de Matemática, Apartado 3008, 3001-501

Coimbra, Portugal

Tim Van der Linden tim.vanderlinden@uclouvain.be

Institut de Recherche en Mathématique et Physique, Université catholique de Louvain, chemin du cyclotron 2 bte L7.01.02, 1348 Louvain-la-Neuve, Belgium 